\title{
Approximation of function using generalized Zygmund class
}

\author{
H. K. Nigam', Mohammad Mursaleen ${ }^{2,3 *}$ (D) and Supriya Rani ${ }^{1}$
}

\author{
*Correspondence: \\ mursaleenm@gmail.com \\ 2Department of Mathematics, \\ Aligarh Muslim University, Aligarh \\ 202002, India \\ ${ }^{3}$ Department of Medical Research, \\ China Medical University Hospital, \\ China Medical University (Taiwan), \\ Taichung, Taiwan \\ Full list of author information is \\ available at the end of the article
}

\begin{abstract}
In this paper we review some of the previous work done by the earlier authors (Singh et al. in J. Inequal. Appl. 2017:101, 2017; Lal and Shireen in Bull. Math. Anal. Appl. 5(4):1-13, 2013), etc., on error approximation of a function $g$ in the generalized Zygmund space and resolve the issue of these works. We also determine the best error approximation of the functions $g$ and $g^{\prime}$, where $g^{\prime}$ is a derived function of a $2 \pi$-periodic function $g$, in the generalized Zygmund class $X_{z}^{(\eta)}, z \geq 1$, using matrix-Cesàro $\left(T C^{\delta}\right)$ means of its Fourier series and its derived Fourier series, respectively. Theorem 2.1 of the present paper generalizes eight earlier results, which become its particular cases. Thus, the results of (Dhakal in Int. Math. Forum 5(35):1729-1735, 2010; Dhakal in Int. J. Eng. Technol. 2(3):1-15, 2013; Nigam in Surv. Math. Appl. 5:113-122, 2010; Nigam in Commun. Appl. Anal. 14(4):607-614, 2010; Nigam and Sharma in Kyungpook Math. J. 50:545-556, 2010; Nigam and Sharma in Int. J. Pure Appl. Math. 70(6):775-784, 2011; Kushwaha and Dhakal in Nepal J. Sci. Technol. 14(2):117-122, 2013; Shrivastava et al. in IOSR J. Math. 10(1 Ver. I):39-41, 2014) become particular cases of our Theorem 2.1. Several corollaries are also deduced from our Theorem 2.1.

MSC: Primary 42A10; 41A10; secondary 42B05; 42B08

Keywords: Generalized Minkowski inequality (GMI); Best approximation; Generalized Zygmund class; Matrix $(T)$ means; $C^{\delta}$ means; Matrix-Cesàro $(\delta$ order $)\left(T C^{\delta}\right)$; Fourier series (F.S.); Derived Fourier series (D.F.S.)
\end{abstract}

\section{Introduction}

In the past few decades, researchers have been greatly interested in studying the error estimation of functions in different function spaces using summability operators due to their various applications in science and engineering. In this direction, several researchers [11-21] have obtained results on error estimation of functions in different Lipschitz and Hölder classes using different single summability operators. Taking a view point that a product summability operator is more effective than the individual single summability operator, researchers [5, 9, 21-24] have obtained the degree of approximation of functions in different Lipschitz and Hölder classes by different product summability operators.

After reviewing the above mentioned work, we observe that all of the above works cannot provide the best approximation of a function in the function spaces considered. This

(c) The Author(s) 2021. This article is licensed under a Creative Commons Attribution 4.0 International License, which permits use sharing, adaptation, distribution and reproduction in any medium or format, as long as you give appropriate credit to the original author(s) and the source, provide a link to the Creative Commons licence, and indicate if changes were made. The images or other third party material in this article are included in the article's Creative Commons licence, unless indicated otherwise in a credit line to the material. If material is not included in the article's Creative Commons licence and your intended use is not permitted by statutory regulation or exceeds the permitted use, you will need to obtain permission directly from the copyright holder. To view a copy of this licence, visit http://creativecommons.org/licenses/by/4.0/. 
fact strongly motivated us to consider a more advanced class of functions, which can provide the best approximation of a function.

Therefore, in the present work, we establish a theorem on the best error approximation of a function $g$ in the generalized Zygmund class $X_{z}^{(\eta)}(z \geq 1)$ by using the matrix-Cesàro $\left(T C^{\delta}\right)$ product operator of its Fourier series.

In the recent past, researchers have also been greatly interested in studying the derived Fourier series by single or product means. In this direction, Chandra and Dikshit [25] have studied $|B|$ and $|E, q|$ means of derived Fourier series. Lal and Nigam [26] have studied Karamata means of derived Fourier series. Lal and Yadav [27] have considered the $(N, p, q)(C, 1)$ product means of derived Fourier series. However, no one has studied the degree of approximation of functions in any function space by using single or product means of its derived Fourier series. This fact has also motivated us to pursue a study of the degree of approximation of a function in a generalized Zygmund class by the matrixCesàro $\left(T C^{\delta}\right)$ method of its derived Fourier series. Therefore, in this paper, we also establish a theorem on the best error approximation of a function in the generalized Zygmund class $X_{z}^{(\eta)}(z \geq 1)$ by the matrix-Cesàro $\left(T C^{\delta}\right)$ product of its derived Fourier series.

It is important to note that we have considered the $T C^{\delta}$ product operator, which is the most general product operator developed for matrix-Cesàro means.

A separate study of derived Fourier series in the present direction of work is justified, due to its important applications in science and engineering.

In the last six years, investigators $[1,2]$ were working on the error estimation of a function in the $X_{z}^{(\eta)}(z \geq 1)$ space using different summability operators.

In both works $[1,2]$, the second theorem has been proved by considering $\frac{\eta(l)}{l \xi(l)}$ as a nonincreasing function $l$, in addition to the condition that $\frac{\eta(l)}{\xi(l)}$ is nondecreasing, which was considered in their first theorems.

Since the modulus of continuity $\eta$ is a subadditive function, $\frac{\eta(l)}{l}$ is a nonincreasing function of $l$, and the second theorem in each of the above works follows from the first theorem without any additional condition.

Let $\sum_{j=0}^{\infty} d_{j}$ be an infinite series having $j$ th partial sum $s_{j}=\sum_{v=0}^{j} d_{\nu}$. Under usual assumptions on the function $g$, the Fourier series of $g$ is given by

$$
g(y):=\frac{1}{2} a_{0}+\sum_{j=1}^{\infty}\left(a_{j} \cos j y+b_{j} \sin j y\right)
$$

with the $j$ th partial sums $s_{j}(g ; y)$, and the conjugate Fourier series of $g$ is given by

$$
\tilde{s}(g ; y):=\sum_{j=1}^{\infty}\left(a_{j} \sin j y-b_{j} \cos j y\right) .
$$

The series

$$
g^{\prime}(y):=\sum_{j=1}^{\infty} j\left(b_{j} \cos j y-a_{j} \sin j y\right),
$$

which is obtained by differentiating (1) term-by-term, is called the first derived Fourier series of $g(y)$ (Zygmund, [28]). 
Let $T \equiv\left(a_{j, r}\right)$ be an infinite triangular matrix satisfying the conditions of regularity [29], i.e.,

$$
\begin{aligned}
& \sum_{r=0}^{j} a_{j, r}=1 \quad \text { as } j \rightarrow \infty ; \\
& \text { each } r \geq 0 \text { is such that } a_{j, r}=0 \quad \text { as } j \rightarrow \infty ; \\
& \exists N>0 \forall j \geq 0 \text { is such that } \sum_{r=0}^{\infty}\left|a_{j, r}\right|<N .
\end{aligned}
$$

The sequence-to-sequence transformation

$$
\begin{aligned}
t_{j}^{T} & :=\sum_{r=0}^{j} a_{j, r} s_{r} \\
& =\sum_{r=0}^{j} a_{j, j-r} s_{j-r}
\end{aligned}
$$

defines the sequence $t_{j}^{T}$ of triangular matrix means of the sequence $\left\{s_{j}\right\}$, generated by the sequence of coefficients $\left(a_{j, r}\right)$.

If $t_{j}^{T} \rightarrow s$ as $j \rightarrow \infty$, then the infinite series $\sum_{j=0}^{\infty} d_{j}$ or the sequence $\left\{s_{j}\right\}$ is summable to $s$ by the triangular matrix $(T)[28]$.

We write $A_{j}^{0}=s_{j}=\sum_{v=0}^{j} d_{v}, A_{j}^{\delta}=A_{0}^{\delta-1}+A_{1}^{\delta-1}+\cdots+A_{j}^{\delta-1}$, and $E_{j}^{\delta}$ for the value of $A_{j}^{\delta}$ when $a_{0}=1$ and $a_{j}=0$ for $j>0$, i.e., when $A_{j}=1$.

If

$$
\begin{aligned}
C_{j}^{\delta} & =\frac{A_{j}^{\delta}}{E_{j}^{\delta}} \\
& =\frac{1}{E_{j}^{\delta}} \sum_{r=0}^{j} A_{j-r}^{\delta-1} \rightarrow s \quad \text { as } j \rightarrow \infty,
\end{aligned}
$$

where $A_{j}^{\delta}=\sum_{r=0}^{j}\left(\begin{array}{c}j-r+\delta-1 \\ \delta-1\end{array}\right)$ and $E_{j}^{\delta}=\left(\begin{array}{c}j+\delta \\ \delta\end{array}\right)$, then we say that $\sum_{j=0}^{\infty} d_{j}$ or the sequence $\left\{s_{j}\right\}$ is summable to the sum $s$ by $C^{\delta}$ (the Cesàro means of order $\delta$ ) [30].

Superimposing the $T$-method on the $C^{\delta}$ method, $T C^{\delta}$ is obtained. That is, the $T C^{\delta}$ mean of the sequence $\left\{s_{j}\right\}$ is given by

$$
\begin{aligned}
t_{j}^{T C^{\delta}} & :=\sum_{r=0}^{j} a_{j, j-r} C_{j}^{\delta} \\
& =\sum_{r=0}^{j} a_{j, j-r} \frac{1}{A_{j}^{\delta}} \sum_{v=0}^{j} A_{j-\nu}^{\delta-1} s_{v} \\
& =\sum_{r=0}^{j} a_{j, r} \sum_{v=0}^{r} \frac{\left(\begin{array}{c}
r-v+\delta-1 \\
\delta-1
\end{array}\right)}{\left(\begin{array}{c}
r+\delta \\
\delta
\end{array}\right)} s_{v} .
\end{aligned}
$$

If $t_{j}^{T C^{\delta}} \rightarrow s$ as $j \rightarrow \infty$, then $\left\{s_{j}\right\}$ is summable by the $T C^{\delta}$ means to the limit $s$. 
Since the $T$ and $C^{\delta}$ methods are regular, the $T C^{\delta}$ method is also regular. This can be shown as follows:

$$
\begin{aligned}
s_{j} \rightarrow s \quad & \Longrightarrow C_{j}^{\delta} \rightarrow s, \text { as } j \rightarrow \infty \text {, since the } C^{\delta} \text { method is regular } \\
& \Longrightarrow T\left(C_{j}^{\delta}\right)=t_{j}^{T C^{\delta}} \rightarrow s, \text { as } j \rightarrow \infty, \text { since the } T \text { method is regular } \\
& \Longrightarrow\left(T C^{\delta}\right) \text { method is regular. }
\end{aligned}
$$

Remark 1 The $T C^{\delta}$ means reduces to:

(i) $\left(H, \frac{1}{j+1}\right) C^{\delta}$ or $H C^{\delta}$ if $a_{j, r}=\frac{1}{(j-r+1) \log (j+1)}$;

(ii) $(N, p, q) C^{\delta}$ or $N_{p, q} C^{\delta}$ if $a_{j, r}=\frac{p_{j-r} q_{r}}{R_{j}}, R_{j}=\sum_{r=0}^{j} p_{r} q_{j-r} \neq 0$, where $p_{j}$ and $q_{j}$ have their usual meanings;

(iii) $\left(N, p_{j}\right) C^{\delta}$ or $N_{p} C^{\delta}$ if $a_{j, r}=\frac{p_{j-r}}{P_{j}}, P_{j}=\sum_{k r=0}^{j} p_{j} \neq 0, q_{j}=1 \forall j$, where $p_{j}$ has its usual meaning;

(iv) $\left(\tilde{N}, p_{j}\right) C^{\delta}$ or $\tilde{N}_{p} C^{\delta}$ if $a_{j, r}=\frac{p_{r}}{P_{j}}, q_{j}=1 \forall j$, where $q_{j}$ has its usual meaning;

(v) $(E, q) C^{\delta}$ or $E_{q} C^{\delta}$ if $a_{j, r}=\frac{1}{(1+q)}\left(\begin{array}{l}j \\ r\end{array}\right) q^{j-r}$;

(vi) $(E, 1) C^{\delta}$ or $E_{1} C^{\delta}$ if $a_{j, r}=\frac{1}{2^{j}}\left(\begin{array}{l}j \\ r\end{array}\right)$.

Remark 2 In view of Remark $1, T C^{\delta}(\delta=1)$ mean also reduces to $H C^{1}, N_{p, q} C^{1}, N_{p} C^{1}$, $\tilde{N}_{p} C^{1}, E_{q} C^{1}, E_{1} C^{1}$ means.

Example 1 Consider the series

$$
1-10 \sum_{v=1}^{j}(-9)^{v-1}
$$

Then $\left\{s_{j}\right\}$ of (5) is given by

$$
s_{j}=(-9)^{j}
$$

Take

$$
a_{j, r}=\frac{1}{5^{j}}\left(\begin{array}{l}
j \\
r
\end{array}\right) 4^{j-r}
$$

Then

$$
\begin{aligned}
t_{j}^{T} & =\sum_{r=0}^{n} a_{j, r}=a_{j, 0} s_{0}+a_{j, 1} s_{1}+\cdots+a_{j, j} s_{j} \\
& =\frac{1}{5^{j}}\left[\left(\begin{array}{l}
j \\
0
\end{array}\right) 4^{j} \cdot 1-\left(\begin{array}{l}
j \\
1
\end{array}\right) 4^{j-1} \cdot 9+\left(\begin{array}{l}
j \\
2
\end{array}\right) 4^{j-2} \cdot 9^{2}+\cdots+(-1)^{n}\left(\begin{array}{l}
n \\
n
\end{array}\right) 4^{n-n} \cdot 9^{n}\right] \\
& =\frac{1}{5^{j}}(4-9)^{j} \\
& =(-1)^{j} .
\end{aligned}
$$


Here,

$$
(-1)^{j}= \begin{cases}1, & j \text { is even } \\ -1, & j \text { is odd }\end{cases}
$$

We observe that (5) is not summable by $C^{1}$ means.

If $a_{j, r}=\frac{1}{5^{j}}\left(\begin{array}{l}j \\ r\end{array}\right) 4^{j-r}$, then (5) is also not summable by $T$ means. But (5) is summable by $T C^{1}$ means as (6) is summable by $C^{1}$ means. This shows the effectiveness of the product means as compared to single means.

Let $C_{2 \pi}$ denote the Banach space of all $2 \pi$-periodic and continuous functions defined on $[0,2 \pi]$ under the supremum norm $[28]$.

The $j$ th order error approximation of a function $g \in C_{2 \pi}$ is defined by $E_{j}(g):=\inf _{t_{j}}\left\|g-t_{j}\right\|$ where $t_{j}$ is a trigonometric polynomial of degree $j$ [28].

If $E_{j}(g) \rightarrow 0$ as $j \rightarrow \infty$, the $E_{j}(g)$ is said to be the best approximation of $g$ [28].

The $L^{z}$ space is given by

$$
L^{z}[0,2 \pi]:=\left\{g:[0,2 \pi] \mapsto \mathbb{R}: \int_{0}^{2 \pi}|g(y)|^{z} d y<\infty, z \geq 1\right\}
$$

The norm $\|\cdot\|_{r}$ is defined by

$$
\|g\|_{z}:= \begin{cases}\left\{\frac{1}{2 \pi} \int_{0}^{2 \pi}|g(y)|^{z} d y\right\}^{\frac{1}{z}} & \text { for } 1 \leq z<\infty \\ {\operatorname{ess} \sup _{0<y<2 \pi}|g(y)|} \text { for } z=\infty\end{cases}
$$

Let $\eta:[0,2 \pi] \mapsto \mathbb{R}$ be an arbitrary function with $\eta(l)>0$ for $0<l \leq 2 \pi$ and $\lim _{l \rightarrow 0^{+}} \eta(l)=$ $\eta(0)=0$. We define

$$
X_{z}^{(\eta)}:=\left\{g \in L^{z}[0,2 \pi]: 1 \leq z<\infty, \sup _{l \neq 0} \frac{\|g(\cdot+l)+g(\cdot-l)-2 g(\cdot)\|_{z}}{\eta(l)}<\infty\right\}
$$

and

$$
\|g\|_{z}^{(\eta)}:=\|g\|_{z}+\sup _{l \neq 0} \frac{\|g(\cdot+l)+g(\cdot-l)-2 g(\cdot)\|_{z}}{\eta(l)}, \quad z \geq 1 .
$$

Clearly, $\|\cdot\|_{z}^{(\eta)}$ is a norm on $X_{z}^{(\eta)}$.

Hence the Zygmund space $\left(X_{z}^{(\eta)}\right)$ is a Banach space under the norm $\|\cdot\|_{z}^{(\eta)}$. The completeness of $L^{z}(z \geq 1)$ implies the completeness of the space [28]. One can also refer to the papers [31,32] for more details on the Zygmund space.

Remark 3 Throughout the paper, $\eta$ and $\xi$ denote the second order moduli of continuity such that $\frac{\eta(l)}{\xi(l)}$ is positive and nondecreasing in $l$. Then

$$
\|g\|_{z}^{(\xi)} \leq \max \left(1, \frac{\eta(2 \pi)}{\xi(2 \pi)}\right)\|g\|_{z}^{(\eta)}<\infty
$$


Thus,

$$
X_{z}^{(\eta)} \subset X_{z}^{(\xi)} \subset L^{z}, \quad z \geq 1
$$

(Zygmund, [28]).

Remark 4 Necessary and sufficient conditions for a function to be a modulus of continuity of the first order were pointed out by Lebesgue [33] and Nikol'skii [34]. Any modulus of continuity of the first order, $\eta=\eta(g, \cdot)$, satisfies the following conditions:

(i) $\eta(0)=0$;

(ii) the function $\eta$ is continuous on $[0,+\infty)$;

(iii) the function $\eta$ is nondecreasing on $[0,+\infty)$;

(iv) the function $\eta$ is semiadditive, i.e., the inequality $\eta\left(l_{1}+l_{2}\right) \leq \eta\left(l_{1}\right)+\eta\left(l_{2}\right)$ holds for any $l_{1} \geq 0$ and $l_{2} \geq 0$.

Conversely, if a function $\eta$ satisfies $(i)-(i v)$, then it is the first-order modulus of continuity of the function $g(y)=\eta(|y|)$. Moreover, it can be easily seen that $\eta$ is the second order modulus of continuity of the function $g(y)=\frac{\eta(|y|)}{2}$. If a function $\eta$ satisfies conditions $(i)-(i i i)$ and the function $\frac{\eta(l)}{l}$ is nonincreasing on $(0,+\infty)$, then the semiadditivity condition (iv) also holds, and so $\eta$ is the modulus of continuity of the first and second order for some continuous functions.

The second order modulus of continuity satisfies conditions $(i)-(i i i)$ and a further condition, given as follows:

(v) the inequality $\eta(j l) \leq j^{2} \eta(l)$ holds for any $l \geq 0$ and $j \in \mathbb{N}$.

Geit [35] constructed a wide class of functions that are second-order moduli of continuity of $2 \pi$-periodic functions. It can be easily shown that condition $(v)$ for nonnegative functions follows from the following condition:

(vi) the function $\frac{\eta(l)}{l^{2}}$ is nonincreasing on $(0,+\infty)$.

Note 1 Readers may refer to the paper of Konyagin [36] in support of Remark 4. Readers may also refer to the paper of Weiss and Zygmund [37], which dealt with conditions on the second-order modulus of smoothness, sufficient to force absolute continuity of a function. The technique employed in [37] is nearly identical to that of [38].

Remark 5 Therefore, in view of Remark 4 and Note 1, we drop the second theorem established in the papers $[1,2]$, etc., where the condition that $\frac{\eta(l)}{l \xi(l)}$ is a nonincreasing function of $l$, in addition to the condition of their first theorem, is used.

\section{Remark 6}

(i) If we take $\eta(l)=l^{\alpha}$ then $X^{(\eta)}$ reduces to the $X_{\alpha}$ class.

(ii) By taking $\eta(l)=l^{\alpha}, X_{z}^{(\eta)}$ reduces to the $X_{\alpha, z}$ class.

(iii) If $z \rightarrow \infty$ then the $X_{z}^{(\eta)}$ class reduces to the $X^{(\eta)}$ class.

(iv) If we take $z \rightarrow \infty$ then the $X_{z}^{(\eta)}$ class becomes the $X_{\alpha, z}$ class.

(v) Let $0 \leq \beta<\alpha<1$. If $\eta(l)=l^{\alpha}$ and $\xi(l)=l^{\alpha}$, then $\frac{\eta(l)}{\xi(l)}$ is nondecreasing, while $\frac{\eta(l)}{l \xi(l)}$ is a nonincreasing function of $l$.

We write

$$
\phi(y, l)=g(y+l)+g(y-l)-2 g(y)
$$




$$
\begin{aligned}
& h(y, l)=g(y+l)-g(y-l)-2 l g^{\prime}(y), \\
& \Phi(y, l)=\int_{0}^{l}|\phi(u)| d u, \\
& H(y, l)=\int_{0}^{l}|d h(u)|, \\
& \Delta a_{j, r}=a_{j, r}-a_{j, r+1}, \quad 0 \leq r \leq j-1, \\
& K_{j}^{T C^{\delta}}(l)=\frac{1}{2 \pi} \sum_{r=0}^{j} a_{j, j-r} \sum_{v=0}^{r} \frac{\left(\begin{array}{c}
v+\delta-1 \\
\delta-1
\end{array}\right)}{\left(\begin{array}{c}
\delta+r \\
r
\end{array}\right)} \frac{\sin \left(v+\frac{1}{2}\right) l}{\sin \frac{l}{2}} .
\end{aligned}
$$

\section{Theorems}

Theorem 2.1 If $g$ is a $2 \pi$-periodic function belonging to the class $X_{z}^{(\eta)}, z \geq 1$, then the best error estimate of $g$ by the $T C^{\delta}$ method of its F.S. is given by

$$
\left\|t_{j}^{T C^{\delta}}-g\right\|_{z}^{(\xi)}=O\left(\frac{1+\log \pi(j+1)}{\log \pi(j+1)} \int_{\frac{1}{j+1}}^{\pi} \frac{\eta(l)}{l \xi(l)} d l\right)
$$

where $\eta(l)$ and $\xi(l)$ are as defined in Remark 3, provided

$$
\sum_{r=0}^{j-1}\left|\Delta a_{j, r}\right|=O\left(\frac{1}{j+1}\right) \text { and }(j+1) a_{j, j}=O(1) .
$$

Theorem 2.2 If $g^{\prime}$ is a $2 \pi$-periodic function belonging to the class $X_{z}^{(\eta)}$, then the best error estimate of $g^{\prime}$ by the $T C^{\delta}$ method of its D.F.S. is given by

$$
\left\|t_{j}^{T C^{\delta}}-g^{\prime}\right\|_{z}^{(\xi)}=O\left((j+1) \frac{\eta\left(\frac{1}{j+1}\right)}{\xi\left(\frac{1}{j+1}\right)} \int_{0}^{\pi} d h(l)\right),
$$

where $\eta(l)$ and $\xi(l)$ are as defined in Remark 3, provided

$$
\sum_{r=0}^{j-1}\left|\Delta a_{j, r}\right|=O\left(\frac{1}{j+1}\right) \text { and }(j+1) a_{j, j}=O(1) .
$$

\section{Lemmas}

Lemma 3.1 Under the conditions of regularity of matrix $T \equiv\left(a_{j, r}\right)$ for $0<l<\frac{1}{j+1}$,

$$
K_{j}^{T C^{\delta}}(l)=O(j+1)
$$

Proof For $0<l<\frac{1}{j+1}, \sin \frac{l}{2} \geq \frac{l}{\pi}, \sin (j l) \leq j l$ and $\delta>1$, we get

$$
\begin{aligned}
K_{j}^{T C^{\delta}}(l) & =\frac{1}{2 \pi} \sum_{r=0}^{j} a_{j, j-r}\left\{\sum_{v=0}^{r} \frac{\left(\begin{array}{c}
v+\delta-1 \\
\delta-1
\end{array}\right)}{\left(\begin{array}{c}
\delta+r \\
r
\end{array}\right)} \frac{\sin \left(v+\frac{1}{2}\right) l}{\sin \frac{l}{2}}\right\} \\
& \leq \frac{1}{2 \pi} \sum_{r=0}^{j} a_{j, j-r}\left\{\sum_{v=0}^{r} \frac{(v+\delta-1) !}{(\delta-1) ! v !} \frac{\delta ! r !}{(\delta+r) !} \frac{(2 v+1) \frac{l}{2}}{\frac{l}{\pi}}\right\}
\end{aligned}
$$




$$
\begin{aligned}
& =\frac{1}{4} \sum_{r=0}^{j} a_{j, n j-r}\left\{\sum_{v=0}^{r} \frac{(v+\delta-1) !}{(\delta-1) ! v !} \frac{\delta ! r !}{(\delta+r) !}(2 v+1)\right\} \\
& =\frac{1}{4} \sum_{r=0}^{j} a_{j, j-r}\left[\frac{r !}{(\delta+1) \cdots(\delta+r) \delta !} \sum_{v=0}^{r} \frac{(2 v+1)(v+\delta-1) ! \delta !}{v !}\right] \\
& =\frac{1}{4} \sum_{r=0}^{j} a_{j, j-r}\left[\frac{r !}{(\delta+1) \cdots(\delta+r)} \sum_{v=0}^{r} \frac{(2 v+1) \delta(\delta+1) \cdots(\delta+v-1)}{v !}\right] \\
& =\frac{1}{4} \sum_{r=0}^{j} a_{j, j-r}\left[\frac{r !}{(\delta+1) \cdots(\delta+r)}\left\{1+3 \delta+\cdots+\frac{(2 r+1) \delta(\delta+1) \cdots(\delta+r-1)}{r !}\right\}\right] \\
& \leq \frac{1}{4} \sum_{r=0}^{j} a_{j, j-r}\left[\frac{r !}{(\delta+1) \cdots(\delta+r)}\left\{(r+1) \frac{(2 r+1) \delta(\delta+1) \cdots(\delta+r-1)}{r !}\right\}\right] \\
& =\frac{1}{4} \sum_{r=0}^{j} a_{j, j-r}\left[\frac{(r+1)(2 r+1) \delta}{(\delta+r)}\right] \\
& \leq \frac{1}{4} \sum_{r=0}^{n} a_{j, j-r}(2 r+1) \delta \\
& \left.=\frac{1}{4}(2 j+1) \delta \sum_{r=0}^{j} a_{j, j-r}\right] \\
& =O(j+1) . \\
& \operatorname{since~} \sum_{r=0} a_{j, j-r}=1
\end{aligned}
$$

Lemma 3.2 Under the conditions of regularity of matrix $T \equiv\left(a_{j, r}\right)$ for $\frac{1}{j+1} \leq l \leq \pi$,

$$
K_{j}^{T C^{\delta}}=O\left(\frac{1}{l}\right)
$$

Proof For $\frac{1}{j+1} \leq l \leq \pi$, by applying Jordan's lemma and the facts that $\sin \frac{l}{2} \geq \frac{l}{\pi}$, $\sin j l \leq 1$, we get

$$
\begin{aligned}
& K_{j}^{T C^{\delta}}(l)=\frac{1}{2 \pi} \sum_{r=0}^{j} a_{j, j-r}\left\{\sum_{\nu=0}^{r} \frac{\left(\begin{array}{c}
v+\delta-1 \\
\delta-1
\end{array}\right)}{\left(\begin{array}{c}
r+\delta \\
\delta
\end{array}\right)} \frac{\sin \left(v+\frac{1}{2}\right) l}{\sin \frac{l}{2}}\right\} \\
& \leq \frac{1}{2 \pi} \sum_{r=0}^{j} a_{j, j-r}\left\{\sum_{v=0}^{r} \frac{\left(\begin{array}{c}
v+\delta-1 \\
\delta-1
\end{array}\right)}{\left(\begin{array}{c}
r+\delta \\
\delta
\end{array}\right)} \frac{1}{\frac{l}{\pi}}\right\} \\
& =\frac{1}{2 l} \sum_{r=0}^{j} a_{j, j-r}\left\{\sum_{\nu=0}^{r} \frac{\left(\begin{array}{c}
v+\delta-1 \\
\delta-1
\end{array}\right)}{\left(\begin{array}{c}
r+\delta \\
\delta
\end{array}\right)}\right\} \\
& =\frac{1}{2 l} \sum_{r=0}^{j} a_{j, j-r} \sum_{v=0}^{r} \frac{(v+\delta-1) !}{v !(\delta-1) !} \times \frac{\delta ! r !}{(\delta+r) !} \\
& =\frac{1}{2 l} \sum_{r=0}^{j} a_{j, j-r} \frac{r !}{(\delta+1) \cdots(\delta+r) \delta !} \sum_{\nu=0}^{r} \frac{(\nu+\delta-1) ! \delta}{\nu !}
\end{aligned}
$$




$$
\begin{aligned}
& =\frac{1}{2 l} \sum_{r=0}^{j} a_{j, j-r} \frac{r !}{(\delta+1) \cdots(\delta+r)} \sum_{v=0}^{r} \frac{\delta(\delta+1) \cdots(\delta+v-1)}{v !} \\
& =\frac{1}{2 l} \sum_{r=0}^{j} a_{j, j-r} \frac{r !}{(\delta+1) \cdots(\delta+r)}\left[1+\delta+\frac{\delta(\delta+1)}{2 !}+\cdots+\frac{\delta(\delta+1) \cdots(\delta+r-1)}{r !}\right] \\
& \leq \frac{1}{2 l} \sum_{r=0}^{j} a_{j, j-r} \frac{r !}{(\delta+1) \cdots(\delta+r)}\left[(r+1) \times \frac{\delta(\delta+1) \cdots(\delta+r-1)}{r !}\right] \\
& =\frac{1}{2 l} \sum_{r=0}^{j} a_{j, j-r} \frac{(r+1) \delta}{(\delta+r)} \\
& \leq O\left(\frac{1}{l}\right) \sum_{r=0}^{j} a_{j, j-r} \\
& =O\left(\frac{1}{l}\right) \quad \text { since } \sum_{r=0}^{j} a_{j, n j-r}=1 \\
& =O\left(\frac{1}{l}\right) .
\end{aligned}
$$

Lemma 3.3 ([2], p. 8) If $g \in X_{z}^{(\eta)}$ then for $0<l \leq \pi$,

(i) $\|\phi(\cdot, l)\|_{z}=O(\eta(l))$.

(ii) If $\eta(l)$ and $\xi(l)$ are defined as in Remark 6 , then $\|\phi(\cdot+u, l)+\phi(\cdot-u, l)-2 \phi(\cdot, l)\|_{z}=O\left(\xi(|u|) \frac{\eta(l)}{\xi(l)}\right)$.

Lemma 3.4 If $g^{\prime} \in X_{z}^{\eta}$ then for $0<l \leq \pi$,

(i) $\|h(\cdot, l)\|_{z}=O(\eta(l))$.

(ii) If $\eta(l)$ and $\xi(l)$ are defined as in Remark 6, then $\|h(\cdot+u, l)-h(\cdot-u, l)-2 l h(\cdot, l)\|_{z}=O\left(\xi(|u|) \frac{\eta(l)}{\xi(l)}\right)$.

Proof (i) We have

$$
|h(y, l)|=\left|g(y+l)-g(y-l)-2 l h^{\prime}(y)\right| .
$$

Applying Minkowski's inequality (Zygmund [28]), we have

$$
\begin{aligned}
\|h(\cdot, l)\|_{z} & \leq\left\|g(y+l)-g(y-l)-2 l g^{\prime}(y)\right\|_{z} \\
& =O(\eta(l)) .
\end{aligned}
$$

Proof (ii) We have

$$
\begin{aligned}
\left|h(y+u, l)-h(y-u, l)-2 l h^{\prime}(y, l)\right| \leq & \left|g(y+u+l)-h(y+u-l)-2 l^{\prime}(y+u)\right| \\
& +\left|g(y-u+l)-g(y-u-l)-2 l^{\prime}(y-u)\right| \\
& +2 l\left|g^{\prime}(y+l)-g^{\prime}(y-l)-2 l g^{\prime \prime}(y)\right| .
\end{aligned}
$$

Applying Minkowski's inequality (Zygmund [28]), we have

$$
\left\|h(\cdot+u, l)-h(\cdot-u, l)-2 l h^{\prime}(\cdot, l)\right\|_{z} \leq\left\|g(\cdot+u+l)-g(\cdot+u-l)-2 l g^{\prime}(\cdot+u)\right\|_{z}
$$




$$
\begin{aligned}
& \quad+\left\|g(\cdot-u+l)-g(\cdot-u-l)-2 \lg ^{\prime}(\cdot-u)\right\|_{z} \\
& \quad+2 l\left\|g^{\prime}(\cdot+l)-g^{\prime}(\cdot-l)-2 \lg ^{\prime \prime}(\cdot)\right\|_{z} \\
& =O(\eta(l)) .
\end{aligned}
$$

Also,

$$
\begin{aligned}
\left\|h(\cdot+u, l)-h(\cdot-u, l)-2 l h^{\prime}(\cdot, l)\right\|_{z} \leq & \left\|g(\cdot+l+u)-g(\cdot+l-u)-2 l g^{\prime}(\cdot+l)\right\|_{z} \\
& +\left\|g(\cdot-l+u)-g(\cdot-l-u)-2 l g^{\prime}(\cdot-l)\right\|_{z} \\
& +2 l\left\|g^{\prime}(\cdot+u)-g^{\prime}(\cdot-u)-2 l g^{\prime \prime}(\cdot)\right\|_{z} \\
= & O(\eta(u)) .
\end{aligned}
$$

For a positive and nondecreasing function $\xi(l)$ and for $l \leq|u|$, we obtain

$$
\begin{aligned}
\left\|h(\cdot+u, l)-h(\cdot-u, l)-2 l h^{\prime}(\cdot, l)\right\|_{z} & =O(\eta(l)) \\
& =O\left(\xi(l)\left(\frac{\eta(l)}{\xi(l)}\right)\right) \\
& =O\left(\xi(|u|)\left(\frac{\eta(l)}{\xi(l)}\right)\right) .
\end{aligned}
$$

For a positive, nondecreasing function $\frac{\eta(l)}{\xi(l)}$ and for $l \geq|u|$, we have

$$
\frac{\eta(l)}{\xi(l)} \geq \frac{\eta(|u|)}{\xi(|u|)}
$$

Then

$$
\begin{aligned}
\left\|h(\cdot+u, l)-h(\cdot-u, l)-2 l h^{\prime}(\cdot, l)\right\|_{z} & =O(\eta(|u|)) \\
& =O\left(\eta(|u|)\left(\frac{\eta(l)}{\xi(l)}\right)\right) .
\end{aligned}
$$

\section{Proofs of the main theorems}

\subsection{Proof of Theorem 2.1}

Proof Due to [39], $s_{r}(g ; y)$ of $(1)$ is given by

$$
s_{r}(g ; y)-g(y)=\frac{1}{2 \pi} \int_{0}^{\pi} \phi(y, l) \frac{\sin \left(r+\frac{1}{2}\right) l}{\sin \frac{l}{2}} d l, \quad r=0,1,2, \ldots
$$

Then,

$$
\begin{aligned}
& \sum_{r=0}^{j} \frac{\left(\begin{array}{c}
r+\delta-1 \\
\delta-1
\end{array}\right)}{\left(\begin{array}{c}
\delta+j \\
\delta
\end{array}\right)}\left[s_{r}(g ; y)-g(y)\right]=\frac{1}{2 \pi} \int_{0}^{\pi} \phi(y, l) \sum_{r=0}^{j} \frac{\left(\begin{array}{c}
r+\delta-1 \\
\delta-1
\end{array}\right)}{\left(\begin{array}{c}
\delta+j \\
\delta
\end{array}\right)} \frac{\sin \left(r+\frac{1}{2}\right) l}{\sin \frac{l}{2}} d l, \\
& C_{j}^{\delta}(y)-g(y)=\frac{1}{2 \pi} \int_{0}^{\pi} \phi(y, l) \sum_{r=0}^{j} \frac{\left(\begin{array}{c}
r+\delta-1 \\
-1
\end{array}\right)}{\left(\begin{array}{c}
\delta+j \\
\delta
\end{array}\right)} \frac{\sin \left(r+\frac{1}{2}\right) l}{\sin \frac{l}{2}} d l .
\end{aligned}
$$


Now,

$$
\begin{aligned}
t_{j}^{T C^{\delta}}(y)-g(y) & =\sum_{r=0}^{j} a_{j, j-r}\left\{C_{r}^{\delta}(y)-g(y)\right\} \\
& =\frac{1}{2 \pi} \int_{0}^{\pi} \phi(y, l) \sum_{r=0}^{j} a_{j, j-r} \sum_{v=0}^{r} \frac{\left(\begin{array}{c}
v+\delta-1 \\
\delta-1
\end{array}\right)}{\left(\begin{array}{c}
\delta+r \\
\delta
\end{array}\right)} \frac{\sin \left(v+\frac{1}{2}\right) l}{\sin \frac{l}{2}} d l \\
& =\frac{1}{2 \pi} \int_{0}^{\pi} \phi(y, l) \sum_{r=0}^{j} a_{j, j-r} \sum_{v=0}^{r} \frac{\left(\begin{array}{c}
v+\delta-1 \\
\delta-1
\end{array}\right)}{\left(\begin{array}{c}
\delta+r \\
\delta
\end{array}\right)} \frac{\sin \left(v+\frac{1}{2}\right) l}{\sin \frac{l}{2}} d l \\
& =\int_{0}^{\pi} \phi(y, l) K_{j}^{T C^{\delta}}(l) d l .
\end{aligned}
$$

Let

$$
\begin{aligned}
T_{j}(y) & =t_{j}^{T C^{\delta}}(y)-g(y) \\
& =\int_{0}^{\pi} \phi(y, l) K_{j}^{T C^{\delta}}(l) d l .
\end{aligned}
$$

Then,

$$
T_{j}(y+u)+T_{j}(y-u)-2 T_{j}(y)=\int_{0}^{\pi}\{\phi(y+u, l)+\phi(y-u, l)-2 \phi(y, u)\} K_{j}^{T C^{\delta}}(l) d l .
$$

Using the GMI [40], we get

$$
\begin{aligned}
\left\|T_{j}(\cdot+u)+T_{j}(\cdot-u)-2 T_{j}(\cdot)\right\|_{z} \leq & \int_{0}^{\pi}\|\phi(\cdot+u, l)+\phi(\cdot-u, l)-2 \phi(\cdot, l)\|_{z}\left|K_{j}^{T C^{\delta}}(l)\right| d l \\
= & \int_{0}^{\frac{1}{j+1}}\|\phi(\cdot+u, l)+\phi(\cdot-u, l)-2 \phi(\cdot, l)\|_{z}\left|K_{j}^{T C^{\delta}}(l)\right| d l \\
& +\int_{\frac{1}{j+1}}^{\pi}\|\phi(\cdot+u, l)+\phi(\cdot-u, l)-2 \phi(\cdot, l)\|_{z}\left|K_{j}^{T C^{\delta}}(l)\right| d l \\
= & I_{1}+I_{2} .
\end{aligned}
$$

Using Lemmas 3.1 and 3.3 (ii), we obtain

$$
\begin{aligned}
I_{1} & =\int_{0}^{\frac{1}{j+1}}\|\phi(\cdot+u, l)+\phi(\cdot-u, l)-2 \phi(\cdot, l)\|_{z}\left|K_{j}^{T C^{\delta}}(l)\right| d l \\
& =O\left(\int_{0}^{\frac{1}{j+1}} \xi(|u|) \frac{\eta(l)}{\xi(l)}(j+1) d l\right) \\
& =O\left((j+1) \xi(|u|) \int_{0}^{\frac{1}{j+1}} \frac{\eta(l)}{\xi(l)} d l\right) \\
& =O\left((j+1) \xi(|u|) \frac{\eta\left(\frac{1}{j+1}\right)}{\xi\left(\frac{1}{j+1}\right)} \int_{0}^{\frac{1}{j+1}} d l\right) \\
& =O\left(\xi(|u|) \frac{\eta\left(\frac{1}{j+1}\right)}{\xi\left(\frac{1}{j+1}\right)}\right) .
\end{aligned}
$$


Using Lemmas 3.2 and 3.3 (ii), we obtain

$$
\begin{aligned}
I_{2} & =\int_{\frac{1}{j+1}}^{\pi}\|\phi(\cdot+u, l)+\phi(\cdot-u, l)-2 \phi(\cdot, l)\|_{z}\left|K_{j}^{T C^{\delta}}(l)\right| d l \\
& =O\left(\int_{\frac{1}{j+1}}^{\pi} \xi(|u|) \frac{\eta(l)}{\xi(l)} \frac{1}{l} d l\right) \\
& =O\left(\int_{\frac{1}{j+1}}^{\pi} \xi(|u|) \frac{\eta(l)}{l \xi(l)} d l\right) .
\end{aligned}
$$

By (7), (8), and (9), we have

$$
\left\|T_{j}(\cdot+u)+T_{j}(\cdot-u)-2 T_{j}(\cdot)\right\|_{z}=O\left(\xi(|u|) \frac{\eta\left(\frac{1}{j+1}\right)}{\xi\left(\frac{1}{j+1}\right)}\right)+O\left(\int_{\frac{1}{j+1}}^{\pi} \xi(|u|) \frac{\eta(l)}{l \xi(l)} d l\right) .
$$

Thus,

$$
\sup _{u \neq 0} \frac{\left\|T_{j}(\cdot+u)+T_{j}(\cdot-u)-2 T_{j}(\cdot)\right\|_{z}}{\xi(|u|)}=O\left(\frac{\eta\left(\frac{1}{j+1}\right)}{\xi\left(\frac{1}{j+1}\right)}\right)+O\left(\int_{\frac{1}{j+1}}^{\pi} \frac{\eta(l)}{l \xi(l)} d l\right) .
$$

Using Lemmas 3.1, 3.2, and 3.3 (i), we obtain

$$
\begin{aligned}
\left\|T_{j}(\cdot)\right\|_{z} & =\left\|t_{j}^{T C^{\delta}}-g\right\|_{z} \\
& \leq\left(\int_{0}^{\frac{1}{j+1}}+\int_{\frac{1}{j+1}}^{\pi}\right)\|\phi(\cdot, l)\|_{z}\left|K_{j}^{T C^{\delta}}(l)\right| d l \\
& =\int_{0}^{\frac{1}{j+1}}\|\phi(\cdot, l)\|_{z}\left|K_{j}^{T C^{\delta}}(l) d l+\int_{\frac{1}{j+1}}^{\pi}\|\phi(\cdot, l)\|_{z}\right| K_{j}^{T C^{\delta}}(l) d l \\
& =O\left((j+1) \int_{0}^{\frac{1}{j+1}} \eta(l) d l\right)+O\left(\int_{\frac{1}{j+1}}^{\pi} \frac{\eta(l)}{l} d l\right) \\
& =O\left(\eta\left(\frac{1}{j+1}\right)\right)+O\left(\int_{\frac{1}{j+1}}^{\pi} \frac{\eta(l)}{l} d l\right) .
\end{aligned}
$$

We know that

$$
\left\|T_{j}(\cdot)\right\|_{z}^{(\xi)}=\left\|T_{j}(\cdot)\right\|_{z}+\sup _{u \neq 0} \frac{\left\|T_{j}(\cdot+u)+T_{j}(\cdot-u)-2 T_{j}(\cdot)\right\|_{z}}{\xi(|u|)} .
$$

Now, by (10) and (11), we have

$$
\left\|T_{j}(\cdot)\right\|_{z}^{(\xi)}=O\left(\eta\left(\frac{1}{j+1}\right)\right)+O\left(\int_{\frac{1}{j+1}}^{\pi} \frac{\eta(l)}{l} d l\right)+O\left(\frac{\eta\left(\frac{1}{j+1}\right)}{\xi\left(\frac{1}{j+1}\right)}\right)+O\left(\int_{\frac{1}{j+1}}^{\pi} \frac{\eta(l)}{l \xi(l)} d l\right) .
$$

Due to the monotonicity of the function $\xi(l)$,

$$
\eta(l)=\frac{\eta(l)}{\xi(l)} \xi(l) \leq \xi(\pi) \frac{\eta(l)}{\xi(l)}=O\left(\frac{\eta(l)}{\xi(l)}\right) \text { for } 0<l \leq \pi
$$


Hence

$$
O\left(\eta\left(\frac{1}{j+1}\right)\right)=O\left(\frac{\eta\left(\frac{1}{j+1}\right)}{\xi\left(\frac{1}{j+1}\right)}\right) \quad \text { for } l=\frac{1}{j+1}
$$

Again, due to the monotonicity of the function $\xi(l)$,

$$
\int_{\frac{1}{j+1}}^{\pi} \frac{\eta(l)}{l \xi(l)} \xi(l) d l \leq \xi(\pi) \int_{\frac{1}{j+1}}^{\pi} \frac{\eta(l)}{l \xi(l)} d l=O\left(\int_{\frac{1}{j+1}}^{\pi} \frac{\eta(l)}{l \xi(l)} d l\right) .
$$

Thus

$$
\left\|T_{j}(\cdot)\right\|_{z}^{(\xi)}=O\left(\frac{\eta\left(\frac{1}{j+1}\right)}{\xi\left(\frac{1}{j+1}\right)}\right)+O\left(\int_{\frac{1}{j+1}}^{\pi} \frac{\eta(l)}{l \xi(l)} d l\right) .
$$

Using Remark 3, we have

$$
\int_{\frac{1}{j+1}}^{\pi} \frac{\eta(l)}{l \xi(l)} d l \geq \frac{\eta\left(\frac{1}{j+1}\right)}{\xi\left(\frac{1}{j+1}\right)} \int_{\frac{1}{j+1}}^{\pi} \frac{d l}{l} \geq \log \pi(j+1) \frac{\eta\left(\frac{1}{j+1}\right)}{\xi\left(\frac{1}{j+1}\right)}
$$

which gives

$$
\frac{\eta\left(\frac{1}{j+1}\right)}{\xi\left(\frac{1}{j+1}\right)}=O\left[\frac{\int_{\frac{1}{j+1}}^{\pi} \frac{\eta(l)}{l \xi(l)} d l}{\log \pi(j+1)}\right]
$$

By (12) and (13), we have

$$
\left\|T_{j}(\cdot)\right\|_{z}^{(\xi)}=O\left[\frac{\int_{\frac{1}{j+1}}^{\pi} \frac{\eta(l)}{l \xi(l)} d l}{\log \pi(j+1)}\right]+O\left(\int_{\frac{1}{j+1}}^{\pi} \frac{\eta(l)}{l \xi(l)} d l\right) .
$$

Thus,

$$
\left\|t_{j}^{T C^{\delta}}-g\right\|_{z}^{(\xi)}=O\left[\frac{1+\log \pi(j+1)}{\log \pi(j+1)}\left\{\int_{\frac{1}{j+1}}^{\pi} \frac{\eta(l)}{l \xi(l)} d l\right\}\right] .
$$

\section{Proof of Theorem 2.2}

Proof Let $s_{r}(y)$ denote the $r$ th partial sum of the Fourier series (1) given by

$$
s_{r}(g ; y)=\frac{1}{2} a_{0}+\sum_{m=1}^{\infty}\left(a_{m} \cos m y+b_{m} \sin m y\right)
$$

This partial sum can be represented as a definite integral. We have

$$
s_{r}(g ; y)=\frac{1}{2 \pi} \int_{0}^{2 \pi} g(u) d u
$$




$$
\begin{aligned}
& +\frac{1}{\pi} \sum_{m=1}^{r}\left\{\cos m y \int_{0}^{2 \pi} g(u) \cos m u d u+\sin m y \int_{0}^{2 \pi} g(u) \sin m u d u\right\} \\
= & \frac{1}{2 \pi} \int_{0}^{2 \pi}\left\{\frac{1}{2}+\sum_{m=1}^{r} \cos m(y-u)\right\} g(u) d u \\
= & \frac{1}{2 \pi} \int_{0}^{2 \pi} \frac{\sin \left(r+\frac{1}{2}\right)(y-u)}{\sin \frac{1}{2}(y-u)} g(u) d u .
\end{aligned}
$$

Putting $u=y+l$, this becomes

$$
s_{r}(g ; y)=\frac{1}{2 \pi} \int_{-y}^{2 \pi-y} \frac{\sin \left(r+\frac{1}{2}\right) l}{\sin \frac{l}{2}} f(y+l) d l,
$$

since the integrand has the period $2 \pi$, and so takes the same values in $(2 \pi-y, 2 \pi)$ as in $(-y, 0)$.

$$
s_{r}(g ; y)=\frac{1}{2 \pi} \int_{0}^{2 \pi} \frac{\sin \left(r+\frac{1}{2}\right) l}{\sin \frac{l}{2}} f(y+l) d l,
$$

which may also be written in the form

$$
s_{r}(g ; y)=\frac{1}{2 \pi} \int_{0}^{\pi} \frac{\sin \left(r+\frac{1}{2}\right) l}{\sin \frac{l}{2}}\{g(y+l)+g(y-l)\} d l .
$$

Denoting by $s_{r}(y)$ the sum of first $r$-terms of the derived Fourier series (3), we get

$$
\begin{aligned}
s_{r}^{\prime}(g ; y)=\frac{1}{2 \pi} \int_{0}^{\pi}\{g(y+l)+g(y-l)\}\left\{\frac{d}{d l} \frac{\sin \left(r+\frac{1}{2}\right) l}{\sin \frac{l}{2}}\right\} \\
\Longrightarrow s_{r}^{\prime}(g ; y)=\frac{1}{2 \pi} \int_{0}^{\pi} \frac{\sin \left(r+\frac{1}{2}\right) l}{\sin \frac{l}{2}} d\{g(y+l)-g(y-l)\} \\
=\frac{1}{2 \pi} \int_{0}^{\pi} \frac{\sin \left(r+\frac{1}{2}\right) l}{\sin \frac{l}{2}} d h(l)+g^{\prime}(y) .
\end{aligned}
$$

Hence

$$
s_{r}^{\prime}(y)-g^{\prime}(y)=\frac{1}{2 \pi} \int_{0}^{\pi} \frac{\sin \left(r+\frac{1}{2}\right) l}{\sin \frac{l}{2}} d h(l) .
$$

Then

$$
\begin{aligned}
& \sum_{r=0}^{j} \frac{\left(\begin{array}{c}
r+\delta-1 \\
\delta-1
\end{array}\right)}{\left(\begin{array}{c}
\delta+j \\
\delta
\end{array}\right)}\left[s_{r}^{\prime}(g ; y)-g^{\prime}(y)\right]=\frac{1}{2 \pi} \int_{0}^{\pi} h(y, l) \sum_{r=0}^{j} \frac{\left(\begin{array}{c}
r+\delta-1 \\
\delta-1
\end{array}\right)}{\left(\begin{array}{c}
\delta+j \\
\delta
\end{array}\right)} \frac{\sin \left(r+\frac{1}{2}\right) l}{\sin \frac{l}{2}} d h(l), \\
& C_{j}^{\delta}(y)-g^{\prime}(y)=\frac{1}{2 \pi} \int_{0}^{\pi} h(y, l) \sum_{r=0}^{j} \frac{\left(\begin{array}{c}
r+\delta-1 \\
-1
\end{array}\right)}{\left(\begin{array}{c}
\delta+j \\
\delta
\end{array}\right)} \frac{\sin \left(r+\frac{1}{2}\right) l}{\sin \frac{l}{2}} d h(l) .
\end{aligned}
$$


Now,

$$
\begin{aligned}
t_{j}^{T C^{\delta}}(y)-g^{\prime}(y) & =\sum_{r=0}^{j} a_{j, j-r}\left\{C_{r}^{\delta}(y)-g^{\prime}(y)\right\} \\
& =\frac{1}{2 \pi} \int_{0}^{\pi} h(y, l) \sum_{r=0}^{j} a_{j, j-r} \sum_{\nu=0}^{r} \frac{\left(\begin{array}{c}
v+\delta-1 \\
\delta-1
\end{array}\right)}{\left(\begin{array}{c}
r+\delta \\
\delta
\end{array}\right)} \frac{\sin \left(v+\frac{1}{2}\right) l}{\sin \frac{l}{2}} d h(l) .
\end{aligned}
$$

Let

$$
T_{j}^{\prime}(y)=t_{j}^{T C^{\delta}}(y)-g^{\prime}(y)
$$

Then

$$
T_{j}^{\prime}(y+u)+T_{j}^{\prime}(y-u)-2 l T_{j}^{\prime}(y)=\int_{0}^{\pi}\left(h(y+u, l)+h(y-u, l)-2 l h^{\prime}(y, l)\right) K_{j}^{T C^{\delta}}(l) d h(l) .
$$

Using the GMI [40],

$$
\begin{aligned}
& \left\|T_{j}^{\prime}(\cdot+u)+T_{j}^{\prime}(\cdot-u)-2 l T_{j}^{\prime}(\cdot)\right\|_{z} \\
& \leq \int_{0}^{\pi}\|h(\cdot+u, l)+h(\cdot-u, l)-2 \operatorname{lh}(\cdot, l)\|_{z}\left|K_{j}^{T C^{\delta}}(l)\right| d h(l) \\
& =\int_{0}^{\frac{1}{j+1}}\left(\|h(\cdot+u, l)+h(\cdot-u, l)-2 \operatorname{lh}(\cdot, l)\|_{z}\left|K_{j}^{T C^{\delta}}(l)\right|\right) d h(l) \\
& \quad+\int_{\frac{1}{j+1}}^{\pi}\left(\|h(\cdot+u, l)+h(\cdot-u, l)-2 \operatorname{lh}(\cdot, l)\|_{z}\left|K_{j}^{T C^{\delta}}(l)\right|\right) d h(l) \\
& =I_{3}+I_{4} .
\end{aligned}
$$

Using Lemmas 3.1 and 3.4 (ii), we obtain

$$
\begin{aligned}
I_{3} & =\int_{0}^{\frac{1}{j+1}}\|h(\cdot+u, l)+h(\cdot-u, l)-2 l h(\cdot, l)\|_{z}\left|K_{j}^{T C^{\delta}}(l)\right| d h(l) \\
& =O\left(\int_{0}^{\frac{1}{j+1}} \xi(|u|) \frac{\eta(l)}{\xi(l)}(j+1) d h(l)\right) \\
& =O\left((j+1) \xi(|u|) \int_{0}^{\frac{1}{j+1}} \frac{\eta(l)}{\xi(l)} d h(l)\right) \\
& =O\left((j+1) \xi(|u|) \frac{\eta\left(\frac{1}{j+1}\right)}{\xi\left(\frac{1}{j+1}\right)} \int_{0}^{\frac{1}{j+1}} d h(l)\right) .
\end{aligned}
$$

Now, using Lemmas 3.2 and 3.4 (ii), we get

$$
I_{4}=\int_{\frac{1}{j+1}}^{\pi}\|h(\cdot+u, l)+h(\cdot-u, l)-2 \operatorname{lh}(\cdot, l)\|_{z}\left|K_{j}^{T C^{\delta}}(l)\right| d h(l)
$$




$$
\begin{aligned}
& =O\left(\int_{\frac{1}{j+1}}^{\pi} \xi(|u|) \frac{\eta(l)}{\xi(l)} \frac{1}{l} d h(l)\right) \\
& =O\left(\int_{\frac{1}{j+1}}^{\pi} \xi(|u|) \frac{\eta(l)}{l \xi(l)} d h(l)\right) .
\end{aligned}
$$

By (17), (18), and (19), we have

$$
\begin{aligned}
& \left\|T_{j}^{\prime}(\cdot+u)+T_{j}^{\prime}(\cdot-u)-2 l T_{j}^{\prime}(\cdot)\right\|_{z} \\
& \quad=O\left((j+1) \xi(|u|) \frac{\eta\left(\frac{1}{j+1}\right)}{\xi\left(\frac{1}{j+1}\right)}\right) \int_{0}^{\frac{1}{j+1}} d h(l)+O\left(\int_{\frac{1}{j+1}}^{\pi} \xi(|u|) \frac{\eta(l)}{l \xi(l)} d h(l)\right) .
\end{aligned}
$$

Thus,

$$
\begin{aligned}
\sup _{u \neq 0} & \frac{\left\|T_{j}^{\prime}(\cdot+u)+T_{j}^{\prime}(\cdot-u)-2 l T_{j}^{\prime}(\cdot)\right\|_{z}}{\xi(|u|)} \\
= & O\left((j+1) \frac{\eta\left(\frac{1}{j+1}\right)}{\xi\left(\frac{1}{j+1}\right)}\right) \int_{0}^{\frac{1}{j+1}} d h(l)+O\left(\int_{\frac{1}{j+1}}^{\pi} \frac{\eta(l)}{l \xi(l)} d h(l)\right) .
\end{aligned}
$$

Using Lemmas 3.1, 3.2, and 3.4 (i), we obtain

$$
\begin{aligned}
\left\|T_{j}^{\prime}(\cdot)\right\|_{z} & =\left\|t_{j}^{T C^{\delta}}-g^{\prime}\right\|_{z} \\
& \leq\left(\int_{0}^{\frac{1}{j+1}}+\int_{\frac{1}{j+1}}^{\pi}\right)\|h(\cdot, l)\|_{z}\left|K_{j}^{T C^{\delta}}(l)\right| d h(l) \\
& =\int_{0}^{\frac{1}{j+1}}\|h(\cdot, l)\|_{z}\left|K_{j}^{T C^{\delta}}(l) d h(l)+\int_{\frac{1}{j+1}}^{\pi}\|h(\cdot, l)\|_{z}\right| K_{j}^{T C^{\delta}}(l) d h(l) \\
& =O\left((j+1) \int_{0}^{\frac{1}{j+1}} \eta(l) d h(l)\right)+O\left(\int_{\frac{1}{j+1}}^{\pi} \frac{\eta(l)}{l} d h(l)\right) \\
& =O\left((j+1) \eta\left(\frac{1}{j+1}\right) \int_{0}^{\frac{1}{j+1}} d h(l)\right)+O\left(\int_{\frac{1}{j+1}}^{\pi} \frac{\eta(l)}{l} d h(l)\right) .
\end{aligned}
$$

By (21) and (22), we know that

$$
\begin{aligned}
\left\|T_{j}^{\prime}(\cdot)\right\|_{z}^{(\xi)}= & \left\|T_{j}^{\prime}(\cdot)\right\|_{z}+\sup _{u \neq 0} \frac{\left\|T_{j}^{\prime}(\cdot+u)+T_{j}^{\prime}(\cdot-u)-2 l T_{j}^{\prime}(\cdot)\right\|_{z}}{\xi(|u|)}, \\
\left\|T_{j}^{\prime}(\cdot)\right\|_{z}^{(\xi)}= & O\left((j+1) \eta\left(\frac{1}{j+1}\right) \int_{0}^{\frac{1}{j+1}} d h(l)\right)+O\left(\int_{\frac{1}{j+1}}^{\pi} \frac{\eta(l)}{l} d h(l)\right) \\
& +O\left((j+1) \frac{\eta\left(\frac{1}{j+1}\right)}{\xi\left(\frac{1}{j+1}\right)} \int_{0}^{\frac{1}{j+1}} d h(l)\right)+O\left(\int_{\frac{1}{j+1}}^{\pi} \frac{\eta(l)}{l \xi(l)} d h(l)\right) .
\end{aligned}
$$

Due to the monotonicity of the function $\xi(l)$,

$$
\eta(l)=\frac{\eta(l)}{\xi(l)} \xi(l) \leq \xi(\pi) \frac{\eta(l)}{\xi(l)}=O\left(\frac{\eta(l)}{\xi(l)}\right), \quad 0<l \leq \pi .
$$


Hence for $l=\frac{1}{j+1}$,

$$
\eta\left(\frac{1}{j+1}\right)=O\left(\frac{\eta\left(\frac{1}{j+1}\right)}{\xi\left(\frac{1}{j+1}\right)}\right) .
$$

Again, due to the monotonicity of the function $\xi(l)$,

$$
\int_{\frac{1}{j+1}}^{\pi} \frac{\eta(l)}{l \xi(l)} \xi(l) d h(l) \leq \xi(\pi) \int_{\frac{1}{j+1}}^{\pi} \frac{\eta(l)}{l \xi(l)} d h(l)=O\left(\int_{\frac{1}{j+1}}^{\pi} \frac{\eta(l)}{l \xi(l)} d h(l)\right) .
$$

Thus,

$$
\left\|T_{j}^{\prime}(\cdot)\right\|_{z}^{(\xi)}=O\left((j+1) \frac{\eta\left(\frac{1}{j+1}\right)}{\xi\left(\frac{1}{j+1}\right)} \int_{0}^{\frac{1}{j+1}} d h(l)\right)+O\left(\int_{\frac{1}{j+1}}^{\pi} \frac{\eta(l)}{l \xi(l)} d h(l)\right) .
$$

Using Remark 3 and the second mean value theorem,

$$
\int_{\frac{1}{j+1}}^{\pi} \frac{\eta(l)}{l \xi(l)} d h(l) \geq(j+1) \frac{\eta\left(\frac{1}{j+1}\right)}{\xi\left(\frac{1}{j+1}\right)} \int_{\frac{1}{j+1}}^{\pi} d h(l) .
$$

By (23) and (24), we have

$$
\begin{aligned}
\left\|t_{j}^{T C^{\delta}} \cdot-g^{\prime}\right\|_{z}^{(\xi)} & =O\left((j+1) \frac{\eta\left(\frac{1}{j+1}\right)}{\xi\left(\frac{1}{j+1}\right)} \int_{0}^{\frac{1}{j+1}} d h(l)\right)+O\left((j+1) \frac{\eta\left(\frac{1}{j+1}\right)}{\xi\left(\frac{1}{j+1}\right)} \int_{\frac{1}{j+1}}^{\pi} d h(l)\right) \\
& =O\left((j+1) \frac{\eta\left(\frac{1}{j+1}\right)}{\xi\left(\frac{1}{j+1}\right)} \int_{0}^{\pi} d h(l)\right) .
\end{aligned}
$$

\section{Corollaries}

Corollary 6.1 Let $g \in X_{\alpha, z}, z \geq 1$, and $0 \leq \beta<\alpha \leq 1$. Then

$$
E_{j}(g)=\left\|t_{j}^{T C^{\delta}}-g\right\|_{z}^{(\xi)} \|_{(\beta), z}= \begin{cases}O\left[\frac{(1+\log \pi(j+1))(j+1)^{\beta-\alpha}}{\log \pi(j+1)}\right], & 0 \leq \beta<\alpha<1, \\ O\left[\frac{1+\log \pi(j+1)}{(j+1) \log \pi(j+1)}\right], & \beta=0, \alpha=1 .\end{cases}
$$

Proof Taking $\eta(l)=l^{\alpha}, \xi(l)=l^{\beta}, 0 \leq \beta<\alpha<1$ in (14) gives

$$
\left\|t_{j}^{T C^{\delta}}-g\right\|_{(\beta), z}=O\left(\frac{1+\log \pi(j+1)}{\log \pi(j+1)} \int_{\frac{1}{j+1}}^{\pi} l^{\alpha-\beta-1} d l\right) .
$$

Now,

$$
\left\|t_{j}^{T C^{\delta}}-g\right\|_{(\beta), z}= \begin{cases}O\left(\frac{1+\log \pi(j+1)}{\log \pi(j+1)} \int_{\frac{1}{j+1}}^{\pi} l^{\alpha-\beta-1} d l\right), & 0 \leq \beta<\alpha<1, \\ O\left(\frac{1+\pi \log (j+1)}{\log \pi(j+1)} \int_{\frac{1}{j+1}}^{\pi} d l\right), & \beta=0, \alpha=1 .\end{cases}
$$


Therefore,

$$
\left\|t_{j}^{T C^{\delta}}-g\right\|_{z}^{(\xi)} \|_{(\beta), z}= \begin{cases}O\left[\frac{\left(1+\log \pi(j+1)(j+1)^{\beta-\alpha}\right.}{\log \pi(j+1)}\right], & 0 \leq \beta<\alpha<1, \\ O\left[\frac{1+\log \pi(j+1)}{(j+1) \log \pi(j+1)}\right], & \beta=0, \alpha=1 .\end{cases}
$$

Corollary 6.2 Following Remark 1 (i), we obtain

$$
\left\|t_{j}^{H C^{\delta}}-g\right\|_{z}^{(\xi)}=O\left(\frac{1+\log \pi(j+1)}{\log \pi(j+1)} \int_{\frac{1}{j+1}}^{\pi} \frac{\eta(l)}{l \xi(l)} d l\right) .
$$

Corollary 6.3 Following Remark 1 (ii), we obtain

$$
\left\|t_{j}^{N_{p, q} C^{\delta}}-g\right\|_{z}^{(\xi)}=O\left(\frac{1+\log \pi(j+1)}{\log \pi(j+1)} \int_{\frac{1}{j+1}}^{\pi} \frac{\eta(l)}{l \xi(l)} d l\right) .
$$

Corollary 6.4 Following Remark 1 (iii), we obtain

$$
\left\|t_{j}^{N_{p} C^{\delta}}-g\right\|_{z}^{(\xi)}=O\left(\frac{1+\log \pi(j+1)}{\log \pi(j+1)} \int_{\frac{1}{j+1}}^{\pi} \frac{\eta(l)}{l \xi(l)} d l\right) .
$$

Corollary 6.5 Following Remark 1 (iv), we obtain

$$
\left\|t_{j}^{\tilde{N}_{p} C^{\delta}}-g\right\|_{z}^{(\xi)}=O\left(\frac{1+\log \pi(j+1)}{\log \pi(j+1)} \int_{\frac{1}{j+1}}^{\pi} \frac{\eta(l)}{l \xi(l)} d l\right) .
$$

Corollary 6.6 Following Remark 1 (v), we obtain

$$
\left\|t_{j}^{E_{q} C^{\delta}}-g\right\|_{z}^{(\xi)}=O\left(\frac{1+\log \pi(j+1)}{\log \pi(j+1)} \int_{\frac{1}{j+1}}^{\pi} \frac{\eta(l)}{l \xi(l)} d l\right) .
$$

Corollary 6.7 Following Remark 1 (vi), we obtain

$$
\left\|t_{j}^{E_{1} C^{\delta}}-g\right\|_{z}^{(\xi)}=O\left(\frac{1+\log \pi(j+1)}{\log \pi(j+1)} \int_{\frac{1}{j+1}}^{\pi} \frac{\eta(l)}{l \xi(l)} d l\right) .
$$

Corollary 6.8 Following Remark 2, we obtain

$$
\left\|t_{j}^{T C^{1}}-g\right\|_{z}^{(\xi)}=O\left(\frac{1+\log \pi(j+1)}{\log \pi(j+1)} \int_{\frac{1}{j+1}}^{\pi} \frac{\eta(l)}{l \xi(l)} d l\right) .
$$

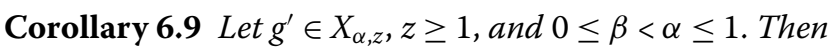

$$
\left\|t_{j}^{T C^{\delta}}-g^{\prime}\right\|_{(\beta), z}= \begin{cases}O\left((j+1)^{\beta-\alpha+1} \int_{0}^{\pi}\right), & 0 \leq \beta<\alpha<1, \\ O\left(\int_{0}^{\pi} d h(l)\right), & \beta=0, \alpha=1 .\end{cases}
$$

Proof Taking $\eta(l)=l^{\alpha}, \xi(l)=l^{\beta}, 0 \leq \beta<\alpha<1$ in (25) yields

$$
\left\|t_{j}^{T C^{\delta}}-g^{\prime}\right\|_{(\beta), z}=O\left((j+1)^{\beta-\alpha+1} \int_{0}^{\pi} d h(l)\right) .
$$


Now,

$$
\left\|t_{j}^{T C^{\delta}}-g^{\prime}\right\|_{(\beta), z}= \begin{cases}O\left((j+1)^{\beta-\alpha+1} \int_{0}^{\pi}\right), & 0 \leq \beta<\alpha<1, \\ O\left(\int_{0}^{\pi} d h(l)\right), & \beta=0, \alpha=1 .\end{cases}
$$

Corollary 6.10 Following Remark 1 (i), we obtain

$$
\left\|t_{j}^{H C^{\delta}}-g^{\prime}\right\|_{z}^{(\xi)}=O\left((j+1) \frac{\eta\left(\frac{1}{j+1}\right)}{\xi\left(\frac{1}{j+1}\right)} \int_{0}^{\pi} d h(l)\right) .
$$

Corollary 6.11 Following Remark 1 (ii), we obtain

$$
\left\|t_{j}^{N_{p, q} C^{\delta}}-g^{\prime}\right\|_{z}^{(v \xi)}=O\left((j+1) \frac{\eta\left(\frac{1}{j+1}\right)}{\xi\left(\frac{1}{j+1}\right)} \int_{0}^{\pi} d h(l)\right) .
$$

Corollary 6.12 Following Remark 1 (iii), we obtain

$$
\left\|t_{j}^{N_{p} C^{\delta}}-g^{\prime}\right\|_{z}^{(\xi)}=O\left((j+1) \frac{\eta\left(\frac{1}{j+1}\right)}{\xi\left(\frac{1}{j+1}\right)} \int_{0}^{\pi} d h(l)\right) .
$$

Corollary 6.13 Following Remark 1 (iv), we obtain

$$
\left\|t_{j}^{\tilde{N}_{p} C^{\delta}}-g^{\prime}\right\|_{z}^{(v \xi)}=O\left((j+1) \frac{\eta\left(\frac{1}{j+1}\right)}{\xi\left(\frac{1}{j+1}\right)} \int_{0}^{\pi} d h(l)\right) .
$$

Corollary 6.14 Following Remark 1 (v), we obtain

$$
\left\|t_{j}^{E_{q} C^{\delta}}-g^{\prime}\right\|_{z}^{(\xi)}=O\left((j+1) \frac{\eta\left(\frac{1}{j+1}\right)}{\xi\left(\frac{1}{j+1}\right)} \int_{0}^{\pi} d h(l)\right) .
$$

Corollary 6.15 Following Remark 1 (vi), we obtain

$$
\left\|t_{j}^{E_{1} C^{\delta}}-g^{\prime}\right\|_{z}^{(\xi)}=O\left((j+1) \frac{\eta\left(\frac{1}{j+1}\right)}{\xi\left(\frac{1}{j+1}\right)} \int_{0}^{\pi} d h(l)\right) .
$$

Corollary 6.16 Following Remark 2, we obtain

$$
\left\|t_{j}^{T C^{1}}-g^{\prime}\right\|_{z}^{(\xi)}=O\left((j+1) \frac{\eta\left(\frac{1}{j+1}\right)}{\xi\left(\frac{1}{j+1}\right)} \int_{0}^{\pi} d h(l)\right) .
$$

\section{Remark 7}

(i) Corollary 6.1 can be further reduced using $T C^{1}$ means in view of Remark 2.

(ii) Corollaries 6.2, 6.3, 6.4, 6.5, 6.6, 6.7 can be further reduced using $H C^{1}, N_{p, q} C^{1}$, $N_{p} C^{1}, \tilde{N} C^{1}, E_{q} C^{1}$, and $E_{1} C^{1}$ means, respectively, in view of Remark 2.

(iii) Corollaries 6.10, 6.11, 6.12, 6.13, 6.14, 6.15 can be further reduced using $H C^{1}$, $N_{p, q} C^{1}, N_{p} C^{1}, \tilde{N}_{p} C^{1}, E_{q} C^{1}$, and $E_{1} C^{1}$ means, respectively, in view of Remark 2. 


\section{Remark 8}

(i) In our Theorem 2.1, if $z \rightarrow \infty$, then the $X_{z}^{(\eta)}$ class becomes the $X^{(\eta)}$ class. Also putting $\eta(l)=l^{\alpha}$ and $\zeta(l)=l^{\beta}$ in our Theorem 2.1, the $X^{(\eta)}$ class turns into the $X_{\alpha}$ class. Then for $\beta=0$, the $X_{\alpha}$ class turns into the $\operatorname{Lip}(\alpha)$ class.

(ii) In our Theorem 2.1, by putting $\eta(l)=l^{\alpha}, \zeta(l)=l^{\beta}$ in the $X_{z}^{(\eta)}$ class, the $X_{z}^{(\eta)}$ class turns into the $X_{\alpha, z}$ class. Then for $\beta=0$, the $X_{\alpha, z}$ class turns into the $\operatorname{Lip}(\alpha, z)$ class.

\section{Remark 9}

(i) If $\zeta(l)=l^{\alpha}$ and $z \rightarrow \infty$, then the $\operatorname{Lip}(\zeta(l), z)$ class turns into the $\operatorname{Lip}(\alpha)$ class and thus, the results of $[6,8]$, and [10] reduce to those for the $\operatorname{Lip}(\alpha)$ class.

(ii) If $\beta=0, \zeta(l)=l^{\alpha}$ and $z \rightarrow \infty$, then the $W\left(L_{z}, \zeta(l)\right)$ class turns into the $\operatorname{Lip}(\alpha)$ class. Thus, the results of [5] and [7] reduce to those for the $\operatorname{Lip}(\alpha)$ class.

\section{Particular cases}

(i) Using Remark 8 (i), putting $\delta=1$ in our Theorem 2.1 yields the result of Dhakal [3].

(ii) Using Remark 8 (i), putting $a_{j, r}=\frac{p_{j-r} q_{r}}{R_{j}}$ where $R_{j}=\sum_{r=0}^{j} p_{r} q_{j-r} \neq 0$ and $\delta=1$ in our Theorem 2.1 gives the result of Dhakal [4].

(iii) Using Remark 8 (i) and (ii), putting $a_{j, r}=\frac{1}{2^{j}}\left(\begin{array}{l}j \\ r\end{array}\right)$ and $\delta=1$ in our Theorem 2.1, in view of Remark 9 (ii), the result of Nigam [5] follows.

(iv) Using Remark 8 (i), putting $a_{j, r}=\frac{1}{(1+q)}\left(\begin{array}{l}j \\ r\end{array}\right) q^{j-r}$ and $\delta=1$ in our Theorem 2.1, in view of Remark 9 (i), the result of Nigam [6] follows.

(v) Using Remark 8 (i) and (ii), putting $a_{j, r}=\frac{p_{j-r}}{P_{j}}$ where $P_{j}=\sum_{r=0}^{j} p_{r} \neq 0$ and $\delta=1$ in our Theorem 2.1, in view of Remark 9 (ii), the result of Nigam and Sharma [7] follows.

(vi) Using Remark 8 (i), putting $a_{j, r}=\frac{1}{2^{j}}\left(\begin{array}{r}j \\ r\end{array}\right)$ and $\delta=1$ in our Theorem 2.1, in view of Remark 9 (i), the result of Nigam and Sharma [8] follows.

(vii) Using Remark 8 (ii), putting $a_{j, r}=\frac{p_{j-r} q_{r}}{R_{j}}$ where $R_{j}=\sum_{r=0}^{j} p_{r} q_{j-r} \neq 0$ and $\delta=1$ in our Theorem 2.1, the result of Kushwaha and Dhakal [9] follows.

(viii) Using Remark 8 (i), putting $\delta=1$ in our Theorem 2.1, in view of Remark 9 (i), the result of Shrivastava, Rathore, and Shukla [10] follows.

\section{Conclusion}

In this paper, we have determined the best error approximation of the functions $g$ and $g^{\prime}$, where $g^{\prime}$ is a derived function of a $2 \pi$-periodic function $g$ in the generalized Zygmund class $X_{z}^{(\eta)}, z \geq 1$, using matrix-Cesàro $\left(T C^{\delta}\right)$ means of its Fourier series and its derived Fourier series, respectively. We have proved Theorem 2.1 which generalizes several earlier results, and the results of [3-10] become particular cases of our Theorem 2.1. Several corollaries are also deduced from our Theorem 2.1.

\section{Acknowledgements}

Not applicable.

Funding

No funding available.

Availability of data and materials

Data sharing is not applicable to this article as no datasets were generated or analyzed during the current study. 
Authors' contributions

The authors contributed equally and significantly in writing this paper. All authors read and approved the final manuscript.

\section{Author details}

'Department of Mathematics, Central University of South Bihar, Gaya, 824236, India. ${ }^{2}$ Department of Mathematics, Aligarh Muslim University, Aligarh 202002, India. ${ }^{3}$ Department of Medical Research, China Medical University Hospital, China Medical University (Taiwan), Taichung, Taiwan.

\section{Publisher's Note}

Springer Nature remains neutral with regard to jurisdictional claims in published maps and institutional affiliations.

Received: 21 July 2020 Accepted: 21 December 2020 Published online: 07 January 2021

\section{References}

1. Singh, M.V., Mittal, M.L., Rhoades, B.E.: Approximation of functions in the generalized Zygmund class using Hausdorff means. J. Inequal. Appl. 2017, 101 (2017)

2. Lal, S., Shireen: Best approximation of function of generalized Zygmund class matrix-Euler summability means of Fourier series. Bull. Math. Anal. Appl. 5(4), 1-13 (2013)

3. Dhakal, B.P.: Approximation of functions belonging to the Lip $\alpha$ class by matrix-Cesáro summability method. Int. Math. Forum 5(35), 1729-1735 (2010)

4. Dhakal, B.P.: Approximation of a function $f$ belonging to Lip class by $(N, p, q) C_{1}$ means of its Fourier series. Int. J. Eng. Technol. 2(3), 1-15 (2013)

5. Nigam, H.K.: Degree of approximation of functions belonging to Lip $\alpha$ class and weighted $\left(L_{r}, \xi(t)\right)$ class by product summability method. Surv. Math. Appl. 5, 113-122 (2010)

6. Nigam, H.K.: On degree of approximation of a function belonging to $\operatorname{Lip}(\xi(t), r)$ class by $(E, q)(C, 1)$ product means of Fourier series. Commun. Appl. Anal. 14(4), 607-614 (2010)

7. Nigam, H.K., Sharma, A.: On approximation of functions belonging to $L i p(\alpha, r)$ class and to weighted $W\left(L_{r}, \xi(t)\right)$ class by product means. Kyungpook Math. J. 50, 545-556 (2010)

8. Nigam, H.K., Sharma, K.: Degree of approximation of a function belonging to $\operatorname{Lip}(\xi(t), r)$ class by $(E, 1)(C, 1)$ product means. Int. J. Pure Appl. Math. 70(6), 775-784 (2011)

9. Kushwaha, J.K., Dhakal, B.P.: Approximation of a function belonging to Lip $(\alpha, r)$ class by $N_{p, q}$. $C_{1}$ summability method of its Fourier series. Nepal J. Sci. Technol. 14(2), 117-122 (2013)

10. Shrivastava, U.K., Rathore, C.S., Shukla, S.: Approximation of function belonging to the Lip $(\psi(t), p)$ class by matrix-Cesáro summability method. IOSR J. Math. 10(1 Ver. I), 39-41 (2014)

11. Rhaodes, B.E.: On the degree of approximation of functions belonging to Lipschitz class by Hausdorff means of its Fourier series. Tamkang J. Math. 34(3), 245-247 (2003)

12. Sahney, B.N., Goel, D.S.: On the degree of continuous functions. Ranchi Univ. Math. J. 4, 50-53 (1973)

13. Alexits, G.: Convergence Problems of Orthogonal Series. International Series of Monograms in Pure and Applied Mathematics, vol. 20 (1961). Translated from German by I. Folder

14. Khan, H.H.: On degree of approximation of function belonging to the class Lip $(\alpha, p)$. Indian J. Pure Appl. Math. 5(3), 132-136 (1971)

15. Tiwari, K.S., Bariwal, C.S.: The degree of approximation of functions in the Hólder metric by triangular matrix method of Fourier series. Int. J. Pure Appl. Math. 76(2), 227-232 (2012)

16. Qureshi, K.: On the degree of approximation of a function belonging to the class Lip $\alpha$. Indian J. Pure Appl. Math. 13(8), 898-903 (1982)

17. Qureshi, K.: On the degree of approximation of a periodic function $f$ by almost Nórlund means. Tamkang J. Math. 12(1), 35-38 (1981)

18. Qureshi, K., Neha, H.K.: A class of functions and their degree of approximation. Ganita 41(1), 37-42 (1990)

19. Leindler, L.: Trigonometric approximation of functions in $L_{p}$ norm. J. Math. Anal. Appl. 302, 129-136 (2005)

20. Chandra, P.: Trigonometric approximation of functions in $L_{p}$ norm. J. Math. Anal. Appl. 275(1), 13-26 (2002)

21. Lal, S., Mishra, A.: The method of summation $(E, 1)\left(N, p_{n}\right)$ and trigonometric approximation of function in generalized Hölder metric. J. Indian Math. Soc. 80(1-2), 87-98 (2013)

22. Nigam, H.K.: Degree of approximation of a function belonging to weighted $\left(L_{r}, \xi(t)\right)$ class by $(C, 1)(E, q)$ means. Tamkang J. Math. 42(1), 31-37 (2011)

23. Lal, S.: Approximation of functions belonging to the generalized Lipschitz class by $C^{1} . N_{p}$ summability method of Fourier series. Appl. Math. Comput. 209, 346-350 (2009)

24. Lal, S., Kushwaha, J.K.: Degree of approximation of Lipschitz function by product summability method. Int. Math. Forum 4(43), 2101-2107 (2009)

25. Chandra, P., Dikshit, G.D.: On the $|B|$ and $|E, q|$ summability of a Fourier series, its conjugate series and their derived series. Indian J. Pure Appl. Math. 12(11), 1350-1360 (1981)

26. Lal, S., Nigam, H.K.: On K K -summability of derived Fourier series. Ultra Sci. Phys. Sci. 12(2), 198-203 (2000)

27. Lal, S., Yadav, P.: $(N, p, q)(C, 1)$ summability of derived series of a Fourier series. Bull. Col. Math. Soc. 93(2), 85-92 (2001)

28. Zygmund, A.: Trigonometric Series, 3rd rev. edn. Cambridge University Press, Cambridge (2002)

29. Toeplitz, O.: Überallgemeine lineare Mittelbildungen. Pr. Mat.-Fiz. 22, 113-119 (1913)

30. Hardy, G.H.: Divergent Series. Oxford University Press, London (1949)

31. Nigam, H.K., Mursaleen, M., Rani, S.: Approximation of functions in generalized Zygmund class by double Hausdorff matrix. Adv. Differ. Equ. 2020, 317 (2020)

32. Jiang, Z.-J.: On Stević-Sharma operator from the Zygmund space to the Bloch-Orlicz space. Adv. Differ. Equ. 2015 $228(2015)$ 
33. Lebesgue, H.: Sur la représentation trigonometric approchée des fonctions satisfaisant à une condition de Lipschitz. Bull. Soc. Math. Fr. 38, 184-210 (1910)

34. Nikol'skii, S.M.: Fourier series of functions with a given modulus of continuity. Dokl. Akad. Nauk SSSR 52(3), 191-194 (1946)

35. Geit, V.É.: On functions which are the second modulus of continuity. Izv. Vysš. Učebn. Zaved., Mat. 9, 38-41 (1998) [Russ. Math. 42(9), 36-38 (1998)]

36. Konyagin, S.V.: On the second moduli of continuity. Proc. Steklov Inst. Math. 269, 143-145 (2010)

37. Weiss, M., Zygmund, A.: A note on smooth functions. Indag. Math. 21, 52-58 (1959)

38. Zygmund, A.: A remark on the integral modulus of continuity. Rev. Univ. Nac. Tucumán 7, 259-269 (1950)

39. Titchmarsh, E.C.: The Theory of Functions, 2nd edn. Oxford University Press, London (1939). p. 403

40. Chui, C.K.: An Introduction to Wavelets. Wavelet Analysis and Its Applications, vol. 1. Academic Press, San Diego (1992)

Submit your manuscript to a SpringerOpen ${ }^{\circ}$ journal and benefit from:

- Convenient online submission

- Rigorous peer review

- Open access: articles freely available online

- High visibility within the field

- Retaining the copyright to your article

Submit your next manuscript at $\gg$ springeropen.com 